Review Article

\title{
Curcumin and Resveratrol as Promising Natural Remedies with Nanomedicine Approach for the Effective Treatment of Triple Negative Breast Cancer
}

\author{
Amol Shindikar, Akshita Singh, Malcolm Nobre, and Saurabh Kirolikar \\ Translational Research Laboratory, Tata Memorial Centre, Advanced Centre for Treatment, \\ Research and Education in Cancer (ACTREC), Navi Mumbai 410210, India \\ Correspondence should be addressed to Amol Shindikar; amolshindikar@gmail.com
}

Received 30 December 2015; Revised 23 March 2016; Accepted 6 April 2016

Academic Editor: Rajendra Mehta

Copyright (C) 2016 Amol Shindikar et al. This is an open access article distributed under the Creative Commons Attribution License, which permits unrestricted use, distribution, and reproduction in any medium, provided the original work is properly cited.

\begin{abstract}
Researchers have made considerable progress in last few decades in understanding mechanisms underlying pathogenesis of breast cancer, its phenotypes, its molecular and genetic changes, its physiology, and its prognosis. This has allowed us to identify specific targets and design appropriate chemical entities for effective treatment of most breast cancer phenotypes, resulting in increased patient survivability. Unfortunately, these strategies have been largely ineffective in the treatment of triple negative breast cancer (TNBC). Hormonal receptors lacking render the conventional breast cancer drugs redundant, forcing scientists to identify novel targets for treatment of TNBC. Two natural compounds, curcumin and resveratrol, have been widely reported to have anticancer properties. In vitro and in vivo studies show promising results, though their effectiveness in clinical settings has been less than satisfactory, owing to their feeble pharmacokinetics. Here we discuss these naturally occurring compounds, their mechanism as anticancer agents, their shortcomings in translational research, and possible methodology to improve their pharmacokinetics/pharmacodynamics with advanced drug delivery systems.
\end{abstract}

\section{Introduction}

Recent advances in the field of oncology have failed to undermine the relentless onslaught of the breast cancer epidemic. With breast cancer accounting for nearly $25 \%$ of annual global burden [1] of cancer, out of which $6-10 \%$ are metastatic at present with systemic recurrences in up to $30 \%$ of even early breast cancers [2,3], the need of the hour is novel treatment strategies. Ground breaking work by Perou et al. [4] and Sørlie et al. [5] on the molecular classification of breast cancer in to distinct subtypes, luminal, HER2 enriched, and basal-like, confirmed the heterogeneity of this disease.

The molecular overlap between basal-like breast cancer and TNBC is close to $80 \%$. TNBC accounts for $15-20 \%$ of all breast cancers [6] and is characterized by the lack of hormone receptor expression as well as absence of overexpression/gene amplification of HER2. TNBCs are characterized by poorly differentiated tumors with higher histological grades and high mitotic/proliferative indices. These cancers metastasize early to visceral organs most commonly the brain and lungs resulting in poor disease-free and overall survival [7]. Certain other distinctive features of TNBC include clustering of cases in premenopausal women with high BMI as well as women of African descent $[8,9]$ and a considerable overlap between BRCA- 1 associated cancers and the triple negative phenotype [10-12]. What makes this clinical entity particularly challenging is the lack of targeted treatment in view of ER/PR/HER2 negativity thus rendering hormonal or anti-HER2 therapy redundant.

Several classes of drugs like VEGF inhibitors, tyrosine kinase inhibitors, and PARP inhibitors have been used either in the adjuvant or in the metastatic setting for TNBCs; however the results have till date been consistently disappointing with either failure to progress to phase II trials or failure to replicate phase I/II results in large phase III clinical trials. 
<smiles>COc1cc(/C=C/C(=O)/C=C(O)/C=C/c2ccc(O)c(OC)c2)ccc1O</smiles>

(a) Curcumin<smiles>CO[13CH3]</smiles>

(b) Trans-resveratrol<smiles>Oc1ccc(/C=C/c2cc(O)cc(O)c2)cc1</smiles>

(c) Cis-resveratrol

Figure 1: Structures of (a) curcumin and (b) Trans- and Cis-resveratrol.

Alternatively, natural compounds and their derivatives have been studied as dietary supplements for prevention of cancer or as new chemical entities to treat cancer [13-15] with resveratrol and curcumin as widely studied natural plant derived compounds for treatment of TNBC [15-17]. There are conflicting evidences on the role of resveratrol in prevention/treatment of TNBC. This disparity is usually observed between in vitro and in vivo studies, wherein all in vitro studies in the last two decades demonstrate antitumor properties of resveratrol in TNBC cell lines, while some, not all, of the in vivo studies do not corroborate with the in vitro results [15]. This disparity has been attributed to various factors in in vivo studies including bioavailability, mode of administration, and efficacy [18]. It is in the light of the abovementioned facts that we will review the two natural plant products and the possibility of using advanced drug delivery systems to offset their limitations as prospective therapies.

\section{Physicochemical, Biological, and Structural Similarities of Resveratrol and Curcumin}

Curcumin and resveratrol share similar biosynthesis pathways in spite of different biological origin. Source of origin is 4 -hydroxycinnamic acid of the shikimate pathway. It is the most commonly employed pathway by plants to synthesize amino acids as well as aromatic/phenolic secondary compounds [19].

Both compounds exhibit similarity in features considering their molecular topography which supports the hypothesis that the targets for absorption or efflux may be shared between them and replace each other's uptake when used in combination. With reference to molecular structures, curcumin and resveratrol show close similarities with presence of several phenolic groups as well as unsaturated carbon chains attached to hydroxyl groups [20-22] (Figure 1).

\section{Curcumin}

Curcumin (diferuloymethane) is an extract of the rhizome of turmeric (Curcuma longa Linn) an Indian traditional medicine [23], exhibiting antiangiogenic, antiproliferative, antitumorigenic, antioxidant, and anti-inflammatory properties in both in vitro and in vivo studies [24]. Curcumin negatively regulates various growth factors, protein kinases, transcription factors, inflammatory cytokines, cell receptors, and other oncogenic proteins. Induction of apoptosis and/or arresting different phases of the cell cycle contribute to the antiproliferative effects of curcumin in cancer cells $[25,26]$. Though the antiproliferative effects of curcumin in human breast cancer cell lines, including ER positive, ER negative, and multidrug resistant cells, are time- and dose-dependent and correlate with curcumin's inhibition of ornithine decarboxylase activity [27], the mechanism of action of curcumin is largely unknown. In a study by $\mathrm{Lv}$ et al., human breast cancer cell lines: MDA-MB-231 (TNBC, basal-like) and MCF-7 (ER+, luminal A) treated with curcumin exhibited antitumor effects by inducing apoptosis [28]. Similarly in another study performed by Sun et al., curcumin inhibited the proliferation of MDA-MB-231 cells with the authors hypothesizing that curcumin acts via the EGFR pathway [29]. Fatty acid-binding protein 5 (FABP5), a possible prognostic marker that negates the effects of retinoic acid (RA) via the FABP5/PPAR $\beta / \delta$ pathway, was shown to be inhibited by curcumin thus sensitizing the RA-resistant TNBC cells to RA mediated growth suppression [30].

Investigation of curcumin as a safe remedy in phase I clinical trial was carried out at doses as high as $12 \mathrm{~g} /$ day but its poor systemic uptake, feeble pharmacokinetics, and rapid multiple biotransformations have made it an unfavourable chemical entity in its free form [31-33]. The use of various food ingredient formulators has also been employed to enhance the absorption and bioreactivity of curcumin [34]. Majumdar et al. have also suggested the use of a chemically 
stable form of curcumin albeit a more toxic analog to treat nongastrointestinal cancers. The authors also suggest that curcumin can be formulated such that it is administered intravenously instead of orally. The synergistic effects of curcumin and resveratrol have been found to inhibit colon cancer, suggesting that resveratrol also known to possess anticancer properties may act as a stabilizing agent for curcumin [35].

\section{Resveratrol}

Resveratrol is a naturally occurring polyphenol that has been reported as a cardioprotective, neuroprotective, chemopreventive agent, along with antiageing properties. Studies have been carried out using in vitro and animal models for studying the action of resveratrol on cancer cells and cancer related pathways. Innumerable in vitro studies exist in the literature on the action of resveratrol in various types of cancers like breast cancer [36-38], skin cancer [39, 40], fibrosarcoma [41], lung cancer [42, 43], gastric and colorectal cancer [44], prostate cancer $[45,46]$, pancreatic cancer $[47,48]$, hepatoma $[49,50]$, neuroblastoma [51], and leukemia [52, 53]. However, replication of these results in successful clinical trials has been hampered by its short half-life, poor water solubility, chemical instability, and low bioavailability when taken orally [54-56] and the overriding challenge in using resveratrol in the clinic is to achieve adequate bioavailability at tolerable dose. Drug delivery systems which direct the drugs to specific sites in the body by linking particulate systems or macromolecular carriers to monoclonal antibodies or cell specific ligands are one of the probable methods to enhance the delivery of resveratrol to achieve a therapeutic range [57].

Resveratrol acts in all three stages, that is, initiation, promotion, and progression, which affects the overall process of carcinogenesis. It promotes the cancer cells to undergo apoptosis mediated by Fas/Fas ligand, cyclin-dependent kinases cdk 1 and 2, p53, and cyclins A and B1 [58]. It arrests the cell cycle as a result of irreparable cell DNA damage of cancerous cell [59]. Resveratrol also exerts antiangiogenic property and inhibits the matrix metalloproteinases enzymes, which catalyzes the process of cancerous invasion into deeper tissues [60]. Resveratrol is involved in transcription factor NF- $\kappa \mathrm{B}$ modulation [61], as well as cytochrome P450 isoenzyme CYP1A1 inhibition [62]. It has also been reported that it produces synergistic activity when given in combination with anthracycline derivatives such as doxorubicine, while treating various types of cancers [63].

\section{Bioavailability and Pharmacokinetics of Curcumin and Resveratrol as Polyphenols}

Bioavailability is the rate and extent of absorption of drug at the site of action. When curcumin and resveratrol are given orally, they exhibit poor bioavailability because of short half-life and rapid elimination. To increase high intracellular uptake, large doses need to be administered, which reduces their use as supplements [64-66].
Absorption of most polyphenols does not take place in their native form [67]. These compounds undergo hydrolysis either by colonic microflora or by intestinal enzymes before their absorption. Polyphenols undergo extensive modifications during absorption process; they undergo conjugation process in intestinal cells followed by glucuronidation, methylation, and/or sulfation in the liver $[68,69]$. The chemical structure rather than the concentration of polyphenols determines their bioavailability and nature of metabolite circulating in plasma. Therapeutic potency of polyphenols differs from one to another. After the process of metabolism, polyphenols produce different metabolites compared to their parental forms and circulate in the blood as well as getting absorbed in the tissues. Evaluation of potency of every metabolites is difficult [70].

5.1. Curcumin. Metabolism of large part of curcumin in rats via oral route was reported to be the first biodistribution study [71]. It was shown that metabolism of curcumin occurs mainly in liver [71-73]. Glucuronides of tetrahydrocurcumin (THC) and hexahydrocurcumin (HHC) were investigated as the major metabolites of curcumin in rats by Holder et al. Dihydroferulic acid along with ferulic acid in traces was considered as minor metabolite [74]. Urine of the rats treated with curcumin also showed the presence of sulfate conjugates in addition to glucuronides [75]. Findings of Pan et al. reported that $99 \%$ of curcumin conjugates present in plasma were glucuronides because of glucuronidase catalyzed hydrolysis. The same study finally concluded that curcumin produces major metabolites as tetrahydrocurcumin- (THC-) glucuronoside, dihydrocurcumin-glucuronoside in vivo [76].

In another study conducted on healthy humans, pharmacokinetics of a curcumin preparation in healthy human volunteers was examined at 0.25 to $72 \mathrm{hr}$ after a single oral dose. Given doses of curcumin were $10 \mathrm{~g}(n=6)$ and $12 \mathrm{~g}(n=$ 6). When serum sample of subjects was analyzed using HPLC (50 ng/mL as limit of detection) as an analytical technique, free curcumin was detected in one subject only at any of the 14 time points. However, samples of all subjects were detected with the presence of curcumin glucuronides and sulfates. Plasma samples were not detected with free curcumin from any other subject [77].

5.2. Resveratrol. Resveratrol (3,4',5-trihydroxy-trans-stilbene) is a stilbene class based polyphenolic compound. Rapid and extensive metabolism of the resveratrol and formation of several different metabolites as resveratrol glucuronides and resveratrol sulfates consequently result in zero bioavailability, when administered orally [78]. In vitro experiments involved treatment and incubation of human hepatocytes, human liver microsomes, and rat hepatocytes. Rats and mice were administered with resveratrol via oral and intraperitoneal routes in vivo studies. When samples of rat urine, mouse serum, human hepatocytes, rat hepatocytes, and human liver microsomes were analyzed by HPLC for resveratrol metabolites using methanolic extracts, trans-resveratrol-3$O$-glucuronide and trans-resveratrol-3-sulfate were detected abundantly in all samples. Structures of these conjugates 
were confirmed on incubation with beta-glucuronidase and sulfatase which releases free resveratrol [79].

In another study, $500 \mathrm{mg}$ of resveratrol immediate-release uncoated caplets was administered to ten subjects. Initially, a starting dose of $1 \mathrm{~g}$ was given followed by sequentially increasing it to $2.5 \mathrm{~g}$ and $5.0 \mathrm{~g}$. After obtaining pharmacokinetic data at $5 \mathrm{~g}$ dose, 10 subjects were given dose of $0.5 \mathrm{~g}$.

Plasma and urine samples showed the presence of two monosulfates, one disulfate, two monoglucuronides, and one glucuronide-sulfate when subjected to HPLC analysis. These findings reconfirm the avid metabolism of resveratrol in humans.

Short half-life, nonretention ability, rapid elimination, and undesirable degradation/biotransformation lead to the low bioavailability of parent molecule of curcumin and resveratrol at the site of action [80].

\section{Tumour Vasculatures and Enhanced Permeability and Retention (EPR) Effect and Its Impact on Advanced Drug Delivery Systems (ADDS)}

Over a period of decade, many types of cancers are being treated by conventional chemotherapy using small molecules. Lack of tumour selectivity results in developing severe adverse side effects; consequently drug doses need to be used in less volumes. Drug efficacy also remains suboptimal [81]. To improve the bioavailability and therapeutic efficacy of hydrophobic drugs, development of site specific tumour targeted chemotherapy is the most effective approach for treating different types of cancers most successfully with ease.

Tumour specific targeting at vascular and tissue level can be achieved by the breakthrough discovery of enhanced permeability and retention (EPR) effect of solid tumours. Pathophysiological and anatomical features of tumor vessels are attributed to the rate and extent of EPR effect in most solid tumours. As a result, many researchers across the globe have been employing the concept of tumor targeting drug delivery following the concept of EPR effect to develop efficient and effective anticancer drugs [82].

Tissues undergoing some pathological conditions result in diseases such as cancer or inflammation exhibits the characteristic abnormal vasculature, which enhances the permeability, retention, and extravasation of macromolecular drugs. Activation of vascular permeability factors as well as deregulated angiogenesis contributes to EPR effect of solid tumours. Important characteristic features of abnormal vasculature include formation of large fenestrations $(300 \mathrm{~nm}$ to $4,700 \mathrm{~nm}$ in size) between the endothelial cells because of its discontinuous features. Activation of proangiogenic and antiangiogenic molecules and imbalance in their expression lead to the formation of large fenestration [83]. Abnormal porous vasculature, compression of the lymphatic vessels as a result of increase in number of cancer cells, and dysfunctional lymphangiogenesis, which causes lymphatic drainage impairment in tumor tissues, are also among other factors.

Interstitial fluid of tumours, which constitutes any constructs or macromolecules including encapsulated drugs or antibodies, is retained for longer period than in normal tissues [84-87] (Figure 2). Healthy tissues do not exhibit EPR effect due to normal vasculature with functional lymphatic drainage (Figure 2).

Favourable alteration of drug pharmacokinetics can be achieved by the ability of macromolecular formulations to extravasate and penetrate the tumours. Encapsulation of drugs protects them from undergoing undesirable metabolism/biotransformation and degradation, which results in plasma half-life prolongation and retention. Macromolecular drugs exhibit different biodistribution pattern compared to free drugs. Macromolecules penetrate the tissues and enter the tumour through the process of endocytosis. A study has supported the hypothesis of EPR effect wherein a liposomal formulation of doxorubicin was administered and doxorubicin induced cardiotoxicity was found to be decreased in the patients [88]. Based on these facts and evidences, it can be considered that advantage of abnormal vasculature may contribute to achieving EPR effect for naturally occurring polyphenols including resveratrol and curcumin as well as other highly hydrophobic, insoluble, unstable, less bioavailable compounds using various compatible ADDS methods.

\section{Advanced Drug Delivery Systems}

To overcome the problems of poor bioavailability and poor pharmacokinetics associated with curcumin and resveratrol, numerous ADDS systems like adjuvants, nanoparticles, liposomes, micelles, phospholipid complexes, dendrimers, nanoemulsions, nanogels, and nanogold are being developed by performing extensive studies.

\subsection{Adjuvants}

7.1.1. Curcumin. Adjuvants are known to have an inhibitory activity on hepatic and intestinal glucuronidation. Use of piperine as an adjuvant with curcumin was administered in rats and healthy human volunteers. $2 \mathrm{~g} / \mathrm{kg}$ of curcumin alone showed a maximum serum curcumin level of 1.35 $(0.23 \mu \mathrm{g} / \mathrm{mL}$ at $0.83 \mathrm{~h})$, whereas concomitant administration of piperine $(20 \mathrm{mg} / \mathrm{kg})$ increased the serum concentration of curcumin for a short period of time with a significant increase in its maximum peak level. A decrease in the elimination half-life and clearance of curcumin resulted in an increase of bioavailability in rats. However, in human volunteers consuming a dose of $2 \mathrm{~g}$ curcumin alone, serum levels were either not detectable or found to be very low and piperine produced a $2000 \%$ increase in bioavailability when administered concomitantly [89].

7.1.2. Resveratrol. It has been known that " $\beta$-glucan" has been used as an adjuvant, drug carrier, or in combination with a drug or compound such as resveratrol by developing drug delivery system to enhance the bioavailability. A study was performed by a laboratory to know the possible combination/synergistic effects of $\beta$-glucan and resveratrol on immune reactions. Significant synergetic effects were reported in all cases, wherein potency of these compounds 


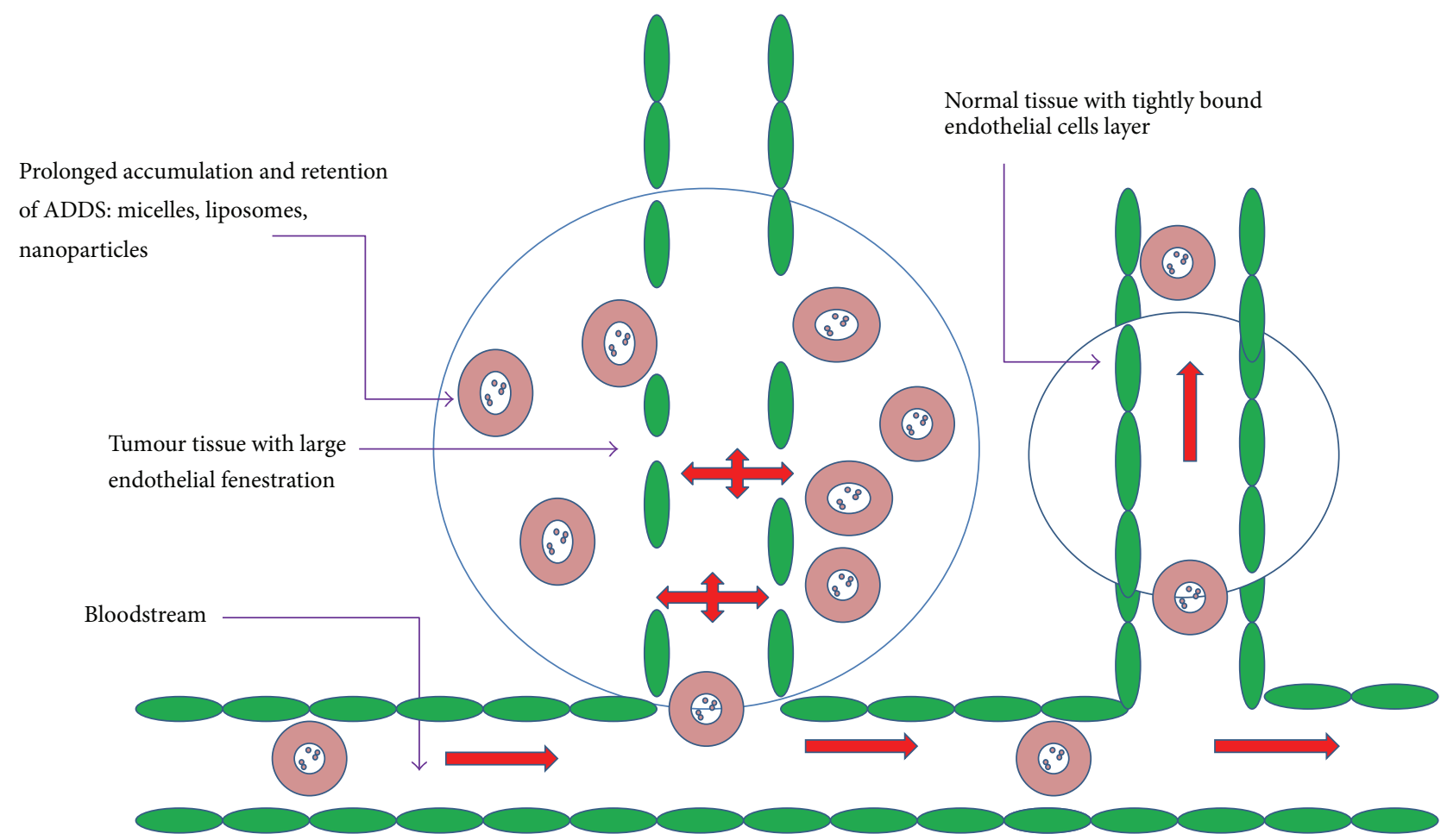

FIGURE 2: Schematic representation of enhanced permeability and retention effect: passive targeting by ADDS (macromolecular extravasation).

on some genes expressions (such as NF- $\kappa \mathrm{B} 2$, Cdc42, and $\mathrm{Bcl}-$ 2 ) in breast cancer cells was tested. Cdc42 levels were found to be upregulated only when resveratrol and glucans were used in combination [90].

7.2. Nanotechnology (Nanoparticles). Nanoparticle technology is a promising drug delivery system developed to enhance the bioavailability of many therapeutic drugs especially highly hydrophobic agents like curcumin (Figure 3(d)).

7.2.1. Curcumin. Limited studies have shown the application of curcumin nanoparticles. Bisht et al. reported the synthesis, physicochemical properties, and cancer related application of "nanocurcumin" (size less than $100 \mathrm{~nm}$ ), a polymer-based nanoparticle of curcumin. In a study it was reported that nanocurcumin showed similar in vitro activity as that of free curcumin in pancreatic cell lines. Nanocurcumin also inhibited activation of the transcription factor $N F-\kappa B$ and reduced steady state levels of proinflammatory cytokines like interleukins and TNF-R similar to free curcumin [91].

Solid lipid nanoparticles (SLNs) which are known as lipid-based drug delivery have become an area of focus in recent times. SLNs as the name suggests are produced by using lipids that are in solid phase at room and body temperatures and are preferred because of their physical stability and ability to protect labile drugs from enzymatic and chemical degradation and controlled release. Curcuminoid having a size of $450 \mathrm{~nm}$ loaded solid lipid nanoparticles (SLNs) was found to be stable for 6 months at room temperature and gave a prolonged and sustained in vitro release of curcuminoids for $12 \mathrm{hrs}$. Additionally, light and oxygen sensitivity of curcuminoids were found to be significantly reduced by formulating the curcuminoids into nanoparticles [92].

7.2.2. Resveratrol. The use of resveratrol in combination with serum albumin in a nanoformulation has been found to significantly inhibit the growth rate of human primary ovarian cancer cells as compared to free resveratrol when implanted subcutaneously [93]. Shao et al. used mPEG poly(epsiloncaprolactone)-based nanoparticles incorporating resveratrol and demonstrated a significantly higher rate of cell death as compared to an equivalent dose of free resveratrol in glioma cells [94]. Incorporating resveratrol in SLN results in decreasing cell proliferation which has been shown to be beneficial in preventing skin cancer [95]. In another study, resveratrol NPs uptake by PCa cell lines was found to be high. In addition, when the PCa, DU-145, and LNCaP were treated with free resveratrol and nanoresveratrol all three cell lines showed significantly elevated cytotoxicity compared to that of free resveratrol at different concentrations (from $10 \mu \mathrm{M}$ to $40 \mu \mathrm{M})$. It proves the consistent sensitivity of nano-RSV towards both the hormone-sensitive LNCaP cells and androgen-independent DU-145 prostate cancer cell lines [96].

7.3. Nanosponges. Nanosponges are nonmutagenic, nonallergenic, and nontoxic and have been used to transport and deliver anticancer drugs. 


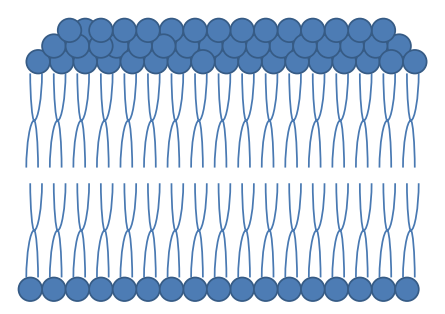

Hydrophilic head

Hydrophobic tail

(a)

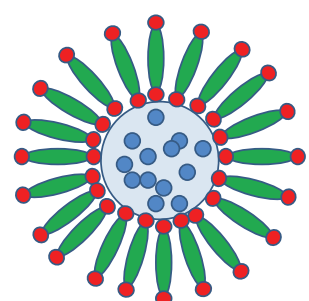

(b)

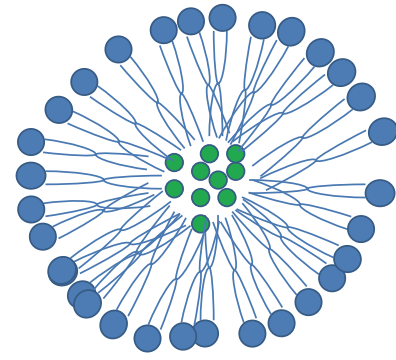

(c)

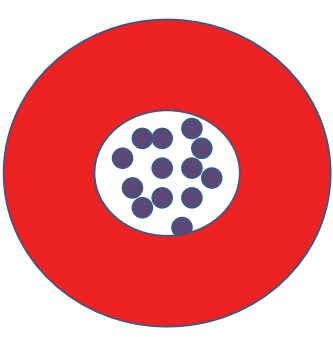

(d)

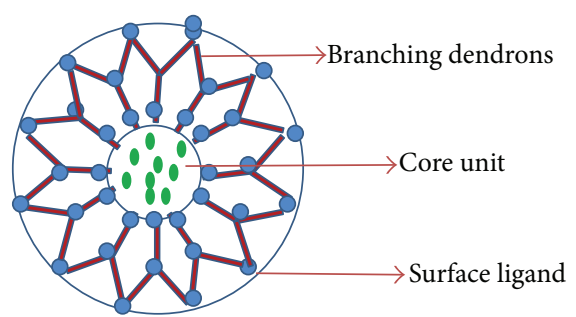

(e)

FIgURE 3: Structures of (a) liposome bilayer, (b) liposome, (c) micelle, (d) polymeric nanoparticles, and (e) dendrimer.

7.3.1. Curcumin. In a study, researchers used cyclodextrinbased nanosponges to enhance curcumin's solubility. They employed dimethyl carbonate as a cross-linker and formulated the complex of $\beta$-cyclodextrin-curcumin nanosponge. The solubilization efficiency of loaded nanosponges was found to be more compared to free curcumin and $\beta$-cyclodextrin complex. Interactions of curcumin with nanosponges were confirmed by the characterization of curcumin nanosponge complex. Also, drug release of curcumin in in vitro studies was well controlled over a prolonged time period and the complex was found to be nonhemolytic [97].

7.3.2. Resveratrol. Cross-linking of different types of cyclodextrin (CD) with cross-linker compound such as carbonyldiimidazole produces nanosponges. They exhibit high solubilization property for the molecules which are poorly soluble in nature. Nanosponges are spherically shaped solid particles [98].

In a study, nanosponges of resveratrol were synthesized. It significantly enhanced the stability as well as solubility of the molecule. Drug permeability was enhanced in in vitro studies on porcine skin and in vivo rabbit buccal mucosa using resveratrol-loaded nanosponges [99].

In another study, nanosponges are nonmutagenic, nonallergenic, and nontoxic and have been used to transport and deliver anticancer drugs [100]. Nanosponges prepared from hyper-cross-linked $\beta$-cyclodextrins have been used as a carrier in in vitro studies with tamoxifen on MCF-7 cells [101]. Similarly William et al. [102] prepared nanosponges from poly(valerolactone-allylvalerolactone) and poly(valerolactone-allylvalerolactone-oxepanedione) and used temozolamide in a drug release study to treat brain tumors in vivo and in vitro. Nanosponges of $\beta$-cyclodextrins carrying paclitaxel were tested for bioavailability and cytotoxicity in vivo in Sprague Dawley rats. As compared to control group, orally administered paclitaxel loaded PLN group showed 3-fold increase in area under the plasma concentration time curve which was found to be significant $(p<0.05)$.

Considering the results obtained from the study, it is evident that the oral bioavailability of paclitaxel can be enhanced using PLN as a most promising new formulation while avoiding the use of cremophor El: Ethanol in Taxol. Similarly, in an in vitro study, when MCF-7 cells were treated with paclitaxel nanosponge complex to determine cytotoxic efficacy, it was observed that cytotoxicity of paclitaxel nanosponge complex was reported to be higher against this cell line as compared to the paclitaxel group $[103,104]$.

7.4. Liposomes. Liposomes carry both hydrophilic and hydrophobic molecules and are known as excellent drug delivery systems (Figures 3(a) and 3(b)).

7.4.1. Curcumin. In vitro and in vivo antitumor activity against human pancreatic carcinoma cells using liposomal curcumin demonstrated that liposomal curcumin suppressed the pancreatic carcinoma growth in xenograft models by inhibiting tumor angiogenesis [105].

In another study, in vitro and in vivo effects of liposomal curcumin on proliferation, apoptosis, signaling, and angiogenesis in human pancreatic carcinoma cells were studied. Results of the study showed NFk-B downregulation, growth 
suppression, and apoptosis induction in vitro. In vivo results reported antitumor and antiangiogenesis activity as well [106].

7.4.2. Resveratrol. In one study, when mitochondrial targeting resveratrol liposomes were used, this induced apoptosis in both nonresistant and resistant cancer cells by dissipating mitochondrial membrane potential. It also increased caspase9 and caspase- 3 activities. Significant antitumor efficacy was exerted by resveratrol liposomes in in xenografted resistant A549/cDDP cancers in nude mice and tumour spheroids by deep penetration [107].

In another study, viability of HEK 293 cells and their photoprotection after UV-B irradiation was tested with free and liposomal resveratrol. Interestingly, cell viability was found to be decreased at $100 \mu \mathrm{M}$ concentration and cell proliferation increased at $10 \mu \mathrm{M}$ and achieved the most effective photoprotection. This study showed effectiveness of resveratrol at $10 \mu \mathrm{M}$ and also toxicity at higher concentrations considering the changes in apoptotic features and cell shape and its detachment [108].

7.5. Polymeric Micelles and Phospholipids Complexes. Micelles and phospholipid complexes can improve the gastrointestinal absorption of natural compounds by decreasing undesirable rapid metabolism and early elimination resulting in higher plasma levels and improved bioavailability (Figure 3(c)).

7.5.1. Curcumin. In vitro model of everted rat intestinal sacs, intestinal absorption of curcumin, and curcumin's micelle with phospholipid as well as bile salt was investigated. In vitro intestinal absorption of curcumin increased from $47 \%$ to $56 \%$ in case of micellar curcumin formulation [109]. Polymeric micellar curcumin pharmacokinetic study showed increase in biological half-life to 60-fold for curcumin in rats compared to curcumin solubilized in a mixture of DMA, PEG, and dextrose [110]. In one study, curcumin $(100 \mathrm{mg} / \mathrm{kg}$ ) and curcumin-phospholipid complex (corresponding to $100 \mathrm{mg} / \mathrm{kg}$ of curcumin) was administered to Sprague-Dawley male rats orally. Maximum plasma curcumin level of $600 \mathrm{ng} / \mathrm{mL}, 2.33$ hours, was detected after oral administration of curcumin-phospholipid complex as opposed to that of free curcumin having maximum plasma concentration of $267 \mathrm{ng} / \mathrm{mL}$ after 1.62 hours of oral dosing with a 1.5-fold increase in the half-life of curcumin [111].

7.5.2. Resveratrol. In a study carried out by Narayanan et al. in which a combination of resveratrol and curcumin was used, a significant decrease of prostatic adenocarcinoma in PTEN knockout mice and in vitro studies on PTEN-CaP8 cancer cells revealed that resveratrol in combination with curcumin inhibited cell growth and induced apoptosis [112].

To increase the effectiveness of resveratrol, drug delivery system like nanocapsules can be used to target drugs at specific sites within the body in the field of cancer biology. The trans-resveratrol-loaded lipid core nanocapsule (RSVLNC) was used to test its antiglioma activity on C6 glioma cell line in vitro and on brain implanted C6 cells in in vivo models. In vitro studies indicated that RSV-LNC decreased the cell viability of $\mathrm{C} 6$ glioma cells to a much greater extent as compared to resveratrol when used alone in solution. In vivo studies RSV-LNC also showed a marked decrease in the size of the tumour, suggesting that RSV-LNC nanocapsules could be used effectively in the treatment of gliomas [113].

7.6. Polymeric Drug Conjugates with Ligands. Ligands are one of the biomarkers that can be used to differentiate between cancer tissue and normal tissue. Attaching ligands to the surface of nanoparticles can help recognize and bind selectively to the receptors that are expressed on tumour cells. This technique will help deliver high doses of anticancer drug directed specifically to the tumour cells sparing the normal cells, thus decreasing the side effects associated with the drug. Inclusion of a targeting antibody or ligand into polymerdrug conjugates has been the most suggested approach to encounter these limitations.

To support this hypothesis, researchers in a study produced a formulation comprising heparin as a carrier and a new folate receptor-targeted paclitaxel nanoparticle (heparin-folate-Taxol (paclitaxel), HFT) and evaluated its activity in nude mouse animal models. Results of this study reported that heparin-folate-Taxol (paclitaxel) showed increased potency against the tumor xenografts growth of human $\mathrm{KB}$ and paclitaxel-resistant $\mathrm{KB}$ derivatives as compared to binary heparin-Taxol or free paclitaxel [114].

In another study, as compared to free paclitaxel treated MCF-7 and MCF-7/Adr cells, transferrin-conjugated paclitaxel loaded (poly(lactic-co-glycolic acid) polymer) nanoparticles showed elevated inhibitory effects on growth of the same cells [115]. In one more study, liposomes conjugated transferrin increased the transfection efficacy of p53, which lead to the ionizing radiation causing sensitization of the transfected cancer cells/xenografts [116]. These studies were performed on the hypothesis based on transferrin as a target for tumor specific drug delivery because it was already investigated that tumor tissues overexpress transferrin receptors as compared to normal tissues [117].

7.7. Dendrimers. In a study, PAMAM encapsulated curcumin and free curcumin were tested on T47D breast cancer cell line for their comparative antiproliferative effect. Using TRAP assay, telomerase activity was studied after $24 \mathrm{hrs}$ of incubation. Inhibitory effect was found to be increased in telomerase activity. No cytotoxicity on cancer cells was found when treated with PAMAM dendrimers encapsulating curcumin. It also showed increase in antiproliferative activity of curcumin [118]. (General representation of dendrimer is as shown in Figure 3(e)).

In another study, several cancer cell lines treated with Curc-OEG inhibition were reported at high level due to apoptosis. Reduction in tumor weights and tumor numbers was also observed when Curc-OEG was intravenously injected in the SKOV-3 tumors xenografted athymic mice as well as subcutaneous (mammary fat pad) MDA-MB-468 tumors. Also no acute and subchronic toxicities in mouse visceral organs at high doses were investigated due to CurcOEG. Doxorubicin and camptothecin anticancer drugs can 
be carried by Curc-OEG nanoparticles as drug carriers to enhance the cytotoxicity in drug resistance cancer cells successfully [119].

7.8. Nanoemulsions. As discussed above, curcumin and resveratrol polyphenolic compounds are lipophilic in nature and have very low solubility in water. As a result of poor solubility, they show lower absorption capacity in the gastrointestinal tract. This leads to limited bioavailability. An effective approach could be nanoencapsulation in o/w nanoemulsions-based delivery systems for incorporating bioactive compounds resveratrol and curcumin.

The lipid droplets are nanometric in size $(50-200 \mathrm{~nm})$ dispersed in hydrophilic phase using a suitable emulsifying agent at the oil/water interface which is known as "nanoemulsions" [120]. A method used as energy-intensive comminution process, using high pressure homogenization, produces a nanoemulsion [121]. Enhancement of passive transport mechanism which is related to concentration gradient across the cell membrane by subcellular size of the nanocapsules leads to improving absorption and bioavailability of resveratrol and curcumin [122]. Authors are conducting several studies in the field of nanocapsulation, extensively on two most promising polyphenols, that is, curcumin [123] and resveratrol, which are known for their many beneficial effects on the human health (anticancer, anti-inflammatory, antimicrobial, antioxidant, and chemopreventive activity), However they face many challenges due to poor bioavailability which limits their clinical use [124]. This study focused on the fabricated stable nanoemulsions using soy lecithin, sugar ester, and modified starch as natural and food acceptable ingredients to encapsulate two polyphenolic compounds, curcumin and resveratrol. This approach is followed for improving their dispersibility in aqueous systems.

At the end of this study, it was observed that nanoemulsion-based delivery systems by encapsulation of polyphenols improved their water dispersibility and protected them from degradation as well as preserving the antioxidant activity. It was also observed that stability of resveratrol was improved when resveratrol $(0.01 \% \mathrm{wt})$ was encapsulated in peanut oilbased nanoemulsions as shown by the significant reduction of the chemical degradation of trans-resveratrol to Cisresveratrol. As far as curcumin $(0.1 \% \mathrm{wt})$ is concerned, it was encapsulated in solid lipid nanoemulsions that trapped the compound in a solid matrix, which lead to improved solubility in aqueous systems and to avoiding the recrystallization and settling of the bioactive compound over time [125].

7.9. Nanogels. Nanogels are crosslinked polymer network ranging size between 10 and $200 \mathrm{~nm}$. In vitro studies were performed on breast cancer, melanoma, and pancreatic cell lines. Cell lines were treated by nanoparticles conjugated curcumin formulation. Results were found to be very interesting. Nanocurcumin increased stability of curcumin, enhanced fluorescence effects, developed bioavailability, improved anticancer effects, got better controlled release, prolonged halflife, and enhanced treatment of melanoma $[126,127]$.

\section{Evidence for Combining Resveratrol and Curcumin}

While evidence for combining resveratrol and curcumin in the treatment of triple negative breast cancer is lacking, in vitro studies have demonstrated synergistic antiproliferative/apoptotic effects in colon [29] and hepatocellular carcinoma [128]. Additionally resveratrol has been shown to enhance both in vivo and in vitro antitumoral effects of curcumin in head and neck carcinoma by increasing the cleavage of PARP-1 and the Bax/Bcl-2 ratio, by inhibiting the phosphorylation of ERK1 and ERK2, and the expression of LC3 II simultaneously with the formation of autophagic vacuoles [129]. More recently resveratrol and curcumin synergistically caused apoptosis in cigarette smoke condensate transformed breast cancer epithelial cells, via p21 mediated inhibition of the Hedgehog-Gli cascade signaling pathway [130]. Coencapsulation of resveratrol and curcumin in lipid core nanocapsules [131] and polymeric micellar codelivery of resveratrol and curcumin [132] have been shown to improve their antioxidant effects and decrease in vitro doxorubicin induced cardiotoxicity, respectively. Despite the lack of more robust evidence for using these two compounds in combination, it would be worthwhile to explore this in future studies.

In one study, wherein resveratrol, curcumin, and quercetin were used as combination therapy ( $200 \mathrm{~nm}$ in size) with or without piperine. affected an in vitro permeability model using apical-to-basal permeability across intact caco2 monolayers. Quercetin, resveratrol, and curcumin were applied apically alone or in combination at $50 \mu \mathrm{M}$ and measured in the basal chamber at $30 \mathrm{~min}$.

Greatest enhancement in permeability was received by resveratrol when combined with other agents: quercetin $(310 \%)$, curcumin $(300 \%)$, and quercetin and curcumin (323\% and $350 \%$ with piperine). Increased permeability was recorded in case of curcumin when combined with quercetin alone (147\%) and both quercetin and resveratrol $(188 \%)$; addition of piperine resulted in a $229 \%$ increase in permeability [133].

\section{Conclusion}

Curcumin and resveratrol appear to be promising anticancer agents but poor solubility, bioavailability, pharmacokinetics, and biodistribution limit their routine use in patients. Rapid elimination, short half-life, undesirable degradation/biotransformation, and instability are the major problems associated with both polyphenols. To overcome these drawbacks, ADDS in conjunction with these drugs present an exciting, novel, and efficient alternative. Evidence for their routine use appears to be limited at present and thorough and extensive studies are mandatory prerequisites prior to testing them on humans. Hence, ADDS has become a common interest and central point for many institutional and pharmaceutical research laboratories across the globe to deliver the drug restricted to the tumour site and reduce the side effects/adverse reactions associated with it.

Considering the literature, we can herby conclude that ADDS is the most promising way to encounter the 
bioavailability and pharmacokinetic issues associated with naturally occurring potential polyphenolic compounds such as curcumin and resveratrol to treat TNBC in most efficient and effective manner.

\section{Competing Interests}

The authors have no conflict of interests.

\section{Authors' Contributions}

(1) Amol Shindikar conceptualized theme and wrote first and final draft, conducted literature search, and designed and finalized paper. (2) Dr. Akshita Singh conducted literature search, critically reviewed and edited first draft, and discussed the critical issues related to final draft. (3) Malcolm Nobre conducted literature search and contributed to first draft for resveratrol studies, proof reading, and corrections in final draft. (4) Saurabh Kirolikar conducted Literature search and discussed the critical issues and corrections in first and final drafts. Amol Shindikar is the first author and Akshita Singh, Malcolm Nobre, and Saurabh Kirolikar are coauthors.

\section{Acknowledgments}

Authors would like to thank Professor Indraneel Mittra for continuous support, motivation, and encouragement while writing the paper.

\section{References}

[1] J. Ferlay, I. Soerjomataram, M. Ervik et al., Cancer Incidence and Mortality Worldwide, IARC Cancer Base no. 11, International Agency for Research on Cancer, Lyon, France, 2013, GLOBOCON, vol. 1, 2012, http://globocan.iarc.fr.

[2] Surveillance, Epidemiology, and End Results (SEER) Program, SEER $*$ Stat Database: Incidence-SEER 9 Regs Public-Use, Nov 2004 Sub (1973-2002), National Cancer Institute, DCCPS, Surveillance Research Program, Cancer Statistics Branch, April 2005, http://www.seer.cancer.gov/.

[3] J. O'Shaughnessy, "Extending survival with chemotherapy in metastatic breast cancer," The Oncologist, vol. 10, no. 3, pp. 2029, 2005.

[4] C. M. Perou, T. Sørile, M. B. Eisen et al., "Molecular portraits of human breast tumours," Nature, vol. 406, no. 6797, pp. 747-752, 2000.

[5] T. Sørlie, C. M. Perou, R. Tibshirani et al., "Gene expression patterns of breast carcinomas distinguish tumor subclasses with clinical implications," Proceedings of the National Academy of Sciences of the United States of America, vol. 98, no. 19, pp. 1086910874, 2001.

[6] C. K. Anders and L. A. Carey, "Biology, metastatic patterns, and treatment of patients with triple-negative breast cancer," Clinical Breast Cancer, vol. 9, supplement 2, pp. S73-S81, 2009.

[7] B. G. Haffty, Q. Yang, M. Reiss et al., "Locoregional relapse and distant metastasis in conservatively managed triple negative early-stage breast cancer," Journal of Clinical Oncology, vol. 24, no. 36, pp. 5652-5657, 2006.

[8] L. Vona-Davis, D. P. Rose, H. Hazard et al., "Triple-negative breast cancer and obesity in a rural appalachian population,"
Cancer Epidemiology Biomarkers and Prevention, vol. 17, no. 12, pp. 3319-3324, 2008.

[9] K. F. Trivers, M. J. Lund, P. L. Porter et al., "The epidemiology of triple-negative breast cancer, including race," Cancer Causes and Control, vol. 20, no. 7, pp. 1071-1082, 2009.

[10] D. P. Atchley, C. T. Albarracin, A. Lopez et al., "Clinical and pathologic characteristics of patients with BRCA-positive and BRCA-negative breast cancer," Journal of Clinical Oncology, vol. 26, no. 26, pp. 4282-4288, 2008.

[11] J. Fasano and F. Muggia, "Breast cancer arising in a BRCAmutated background: therapeutic implications from an animal model and drug development," Annals of Oncology, vol. 20, no. 4, pp. 609-614, 2009.

[12] S. R. Young, R. T. Pilarski, T. Donenberg et al., "The prevalence of BRCA1 mutations among young women with triple-negative breast cancer," BMC Cancer, vol. 9, article 86, 2009.

[13] W. Park, A. R. Amin, Z. G. Chen, and D. M. Shin, "New perspectives of curcumin in cancer prevention," Cancer Prevention Research, vol. 6, no. 5, pp. 387-400, 2013.

[14] R. M. Srivastava, S. Singh, S. K. Dubey, K. Misra, and A. Khar, "Immunomodulatory and therapeutic activity of curcumin," International Immunopharmacology, vol. 11, no. 3, pp. 331-341, 2011.

[15] D. Sinha, N. Sarkar, J. Biswas, and A. Bishayee, "Resveratrol for breast cancer prevention and therapy: preclinical evidence and molecular mechanisms," Seminars in Cancer Biology, 2016.

[16] B. J. Cridge, L. Larsen, and R. J. Rosengren, "Curcumin and its derivatives in breast cancer: current developments and potential for the treatment of drug-resistant cancers," Oncology Discovery, vol. 1, article 6, 2013.

[17] B. C. Litzenburger and P. H. Brown, "Advances in preventive therapy for estrogen-receptor-negative breast cancer," Current Breast Cancer Reports, vol. 6, no. 2, pp. 96-109, 2014.

[18] L. G. Carter, J. A. D’Orazio, and K. J. Pearson, "Resveratrol and cancer: focus on in vivo evidence," Endocrine-Related Cancer, vol. 21, no. 3, pp. R209-R225, 2014.

[19] M. Carocho and I. C. F. R. Ferreira, "The role of phenolic compounds in the fight against cancer-a review," Anti-Cancer Agents in Medicinal Chemistry, vol. 13, no. 8, pp. 1236-1258, 2013.

[20] D. O. Kennedy and E. L. Wightman, "Herbal extracts and phytochemicals: plant secondary metabolites and the enhancement of human brain function," Advances in Nutrition, vol. 2, no. 1, pp. 32-50, 2011.

[21] W. Kopeć, J. Telenius, and H. Khandelia, "Molecular dynamics simulations of the interactions of medicinal plant extracts and drugs with lipid bilayer membranes," FEBS Journal, vol. 280, no. 12, pp. 2785-2805, 2013.

[22] L. G. Korkina, C. De Luca, V. A. Kostyuk, and S. Pastore, "Plant polyphenols and tumors: from mechanisms to therapies, prevention, and protection against toxicity of anti-cancer treatments," Current Medicinal Chemistry, vol. 16, no. 30, pp. 39433965, 2009.

[23] N. Chainani-Wu, "Safety and anti-inflammatory activity of curcumin: a component of tumeric (Curcuma longa)," Journal of Alternative and Complementary Medicine, vol. 9, no. 1, pp. 161168, 2003.

[24] P. Anand, S. G. Thomas, A. B. Kunnumakkara et al., "Biological activities of curcumin and its analogues (Congeners) made by man and Mother Nature," Biochemical Pharmacology, vol. 76, no. 11, pp. 1590-1611, 2008. 
[25] A. Deguchi, "Curcumin targets in inflammation and cancer," Endocrine, Metabolic \& Immune Disorders-Drug Targets, vol. 15, no. 2, supplement 9, pp. 88-96, 2015.

[26] M. K. Shanmugam, G. Rane, M. M. Kanchi et al., "The multifaceted role of curcumin in cancer prevention and treatment," Molecules, vol. 20, no. 2, pp. 2728-2769, 2015.

[27] P. Anand, C. Sundaram, S. Jhurani, A. B. Kunnumakkara, and B. B. Aggarwal, "Curcumin and cancer an 'old-age' disease with an 'age-old' solution," Cancer Letters, vol. 267, no. 1, pp. 133-164, 2008.

[28] Z.-D. Lv, X.-P. Liu, W.-J. Zhao et al., "Curcumin induces apoptosis in breast cancer cells and inhibits tumor growth in vitro and in vivo," International Journal of Clinical \& Experimental Pathology, vol. 7, no. 6, pp. 2818-2824, 2014.

[29] X.-D. Sun, X.-E. Liu, and D.-S. Huang, "Curcumin induces apoptosis of triple-negative breast cancer cells by inhibition of EGFR expression," Molecular Medicine Reports, vol. 6, no. 6, pp. 1267-1270, 2012.

[30] P. Thulasiraman, D. J. McAndrews, and I. Q. Mohiudddin, "Curcumin restores sensitivity to retinoic acid in triple negative breast cancer cells," BMC Cancer, vol. 14, article 724, 2014.

[31] M. Heger, R. F. van Golen, M. Broekgaarden, and M. C. Michel, "The molecular basis for the pharmacokinetics and pharmacodynamics of curcumin and its metabolites in relation to cancers," Pharmacological Reviews, vol. 66, no. 1, pp. 222-307, 2013.

[32] P. Anand, A. B. Kunnumakkara, R. A. Newman, and B. B. Aggarwal, "Bioavailability of curcumin: problems and promises," Molecular Pharmaceutics, vol. 4, no. 6, pp. 807-818, 2007.

[33] M. Bernsdorf, C. Ingvar, L. Jörgensen et al., "Effect of adding gefitinib to neoadjuvant chemotherapy in estrogen receptor negative early breast cancer in a randomized phase II trial," Breast Cancer Research and Treatment, vol. 126, no. 2, pp. 463470, 2011.

[34] B. J. Douglass and D. L. Clouatre, "Beyond yellow curry: assessing commercial curcumin absorption technologies," Journal of the American College of Nutrition, vol. 34, no. 4, pp. 347-358, 2015.

[35] A. P. N. Majumdar, S. Banerjee, J. Nautiyal et al., "Curcumin synergizes with resveratrol to inhibit colon cancer," Nutrition and Cancer, vol. 61, no. 4, pp. 544-553, 2009.

[36] S. Banerjee, C. Bueso-Ramos, and B. B. Aggarwal, "Suppression of 7,12-dimethylbenz(a)anthracene-induced mammary carcinogenesis in rats by resveratrol: role of nuclear factor$\kappa \mathrm{B}$, cyclooxygenase 2, and matrix metalloprotease 9," Cancer Research, vol. 62, no. 17, pp. 4945-4954, 2002.

[37] T. Whitsett, M. Carpenter, and C. A. Lamartiniere, "Resveratrol, but not EGCG, in the diet suppresses DMBA-induced mammary cancer in rats," Journal of Carcinogenesis, vol. 5, article 15, 2006.

[38] Y. Shi, S. Yang, S. Troup et al., "Resveratrol induces apoptosis in breast cancer cells by E2F1-mediated up-regulation of ASPP1," Oncology Reports, vol. 25, no. 6, pp. 1713-1719, 2011.

[39] S. Bhattacharya, S. R. Darjatmoko, and A. S. Polans, "Resveratrol modulates the malignant properties of cutaneous melanoma through changes in the activation and attenuation of the antiapoptotic protooncogenic protein Akt/PKB," Melanoma Research, vol. 21, no. 3, pp. 180-187, 2011.

[40] M. Ndiaye, C. Philippe, H. Mukhtar, and N. Ahmad, “The grape antioxidant resveratrol for skin disorders: promise, prospects, and challenges," Archives of Biochemistry and Biophysics, vol. 508, no. 2, pp. 164-170, 2011.
[41] S.-J. Lee and M.-M. Kim, "Resveratrol with antioxidant activity inhibits matrix metalloproteinase via modulation of SIRT1 in human fibrosarcoma cells," Life Sciences, vol. 88, no. 11-12, pp. 465-472, 2011.

[42] Z. Chen, K. Jin, L. Gao et al., "Anti-tumor effects of bakuchiol, an analogue of resveratrol, on human lung adenocarcinoma A549 cell line," European Journal of Pharmacology, vol. 643, no. 2-3, pp. 170-179, 2010.

[43] L. Whyte, Y.-Y. Huang, K. Torres, and R. G. Mehta, "Molecular mechanisms of resveratrol action in lung cancer cells using dual protein and microarray analyses," Cancer Research, vol. 67, no. 24, pp. 12007-12017, 2007.

[44] M. Sengottuvelan, P. Viswanathan, and N. Nalini, "Chemopreventive effect of trans-resveratrol-a phytoalexin against colonic aberrant crypt foci and cell proliferation in 1,2dimethylhydrazine induced colon carcinogenesis," Carcinogenesis, vol. 27, no. 5, pp. 1038-1046, 2006.

[45] S. H. Mitchell, W. Zhu, and C. Y. F. Young, "Resveratrol inhibits the expression and function of the androgen receptor in LNCaP prostate cancer cells," Cancer Research, vol. 59, no. 23, pp. 5892$5895,1999$.

[46] J. R. Stewart, M. C. Artime, and C. A. O’Brian, "Resveratrol: a candidate nutritional substance for prostate cancer prevention," The Journal of Nutrition, vol. 133, no. 7, supplement, pp. 2440S2443S, 2003.

[47] K. B. Harikumar, A. B. Kunnumakkara, G. Sethi et al., "Resveratrol, a multitargeted agent, can enhance antitumor activity of gemcitabine in vitro and in orthotopic mouse model of human pancreatic cancer," International Journal of Cancer, vol. 127, no. 2, pp. 257-268, 2010.

[48] N. Oi, C.-H. Jeong, J. Nadas et al., "Resveratrol, a red wine polyphenol, suppresses pancreatic cancer by inhibiting leukotriene $\mathrm{A}_{4}$ hydrolase," Cancer Research, vol. 70, no. 23, pp. 9755-9764, 2010.

[49] A. Bishayee, T. Politis, and A. S. Darvesh, "Resveratrol in the chemoprevention and treatment of hepatocellular carcinoma," Cancer Treatment Reviews, vol. 36, no. 1, pp. 43-53, 2010.

[50] T. Mbimba, P. Awale, D. Bhatia et al., "Alteration of hepatic pro-inflammatory cytokines is involved in the resveratrolmediated chemoprevention of chemically-induced hepatocarcinogenesis," Current Pharmaceutical Biotechnology, vol. 13, no. 1, supplement 6, pp. 229-234, 2012.

[51] P. R. van Ginkel, D. Sareen, L. Subramanian et al., "Resveratrol inhibits tumor growth of human neuroblastoma and mediates apoptosis by directly targeting mitochondria," Clinical Cancer Research, vol. 13, pp. 5162-5169, 2007.

[52] J. Dörrie, H. Gerauer, Y. Wachter, and S. J. Zunino, "Resveratrol induces extensive apoptosis by depolarizing mitochondrial membranes and activating caspase- 9 in acute lymphoblastic leukemia cells," Cancer Research, vol. 61, no. 12, pp. 4731-4739, 2001.

[53] M.-F. Tsan, J. E. White, J. G. Maheshwari, and G. Chikkappa, "Anti-leukemia effect of resveratrol," Leukemia \& Lymphoma, vol. 43, no. 5, pp. 983-987, 2002.

[54] J. M. Smoliga and O. Blanchard, "Enhancing the delivery of resveratrol in humans: if low bioavailability is the problem, what is the solution?" Molecules, vol. 19, no. 11, pp. 17154-17172, 2014.

[55] R. Pangeni, J. K. Sahni, J. Ali, S. Sharma, and S. Baboota, "Resveratrol: review on therapeutic potential and recent advances in drug delivery," Expert Opinion on Drug Delivery, vol. 11, no. 8, pp. 1285-1298, 2014. 
[56] J. M. Smoliga, O. Vang, and J. A. Baur, "Challenges of translating basic research into therapeutics: resveratrol as an example," Journals of Gerontology-Series A: Biological Sciences and Medical Sciences, vol. 67, no. 2, pp. 158-167, 2012.

[57] A. C. Santos, F. Veiga, and A. J. Ribeiro, "New delivery systems to improve the bioavailability of resveratrol," Expert Opinion on Drug Delivery, vol. 8, no. 8, pp. 973-990, 2011.

[58] D. A. Benitez, E. Pozo-Guisado, A. Alvarez-Barrientos, P. M. Fernandez-Salguero, and E. A. Castellón, "Mechanisms involved in resveratrol-induced apoptosis and cell cycle arrest in prostate cancer-derived cell lines," Journal of Andrology, vol. 28, no. 2, pp. 282-293, 2007.

[59] A. C. Faber and T. C. Chiles, "Resveratrol induces apoptosis in transformed follicular lymphoma OCI-LY8 cells: evidence for a novel mechanism involving inhibition of BCL6 signaling," International Journal of Oncology, vol. 29, no. 6, pp. 1561-1566, 2006.

[60] Y. Cao, Z.-D. Fu, F. Wang, H.-Y. Liu, and R. Han, "Antiangiogenic activity of resveratrol, a natural compound from medicinal plants," Journal of Asian Natural Products Research, vol. 7, no. 3, pp. 205-213, 2005.

[61] J. Leiro, J. A. Arranz, N. Fraiz, M. L. Sanmartín, E. Quezada, and F. Orallo, "Effect of cis-resveratrol on genes involved in nuclear factor kappa B signaling," International Immunopharmacology, vol. 5, no. 2, pp. 393-406, 2005.

[62] Y. J. Chun, M. Y. Kim, and F. P. Guengerich, "Resveratrol is a selective human cytochrome P450 1A1 inhibitor," Biochemical and Biophysical Research Communications, vol. 262, no. 1, pp. 20-24, 1999.

[63] A. M. Al-Abd, A. M. Mahmoud, G. A. El-Sherbiny et al., "Resveratrol enhances the cytotoxic profile of docetaxel and doxorubicin in solid tumour cell lines in vitro," Cell Proliferation, vol. 44, no. 6, pp. 591-601, 2011.

[64] M. Russo, C. Spagnuolo, I. Tedesco, S. Bilotto, and G. L. Russo, "The flavonoid quercetin in disease prevention and therapy: facts and fancies," Biochemical Pharmacology, vol. 83, no. 1, pp. 6-15, 2012.

[65] C. D. Lao, M. T. Ruffin IV, D. Normolle et al., "Dose escalation of a curcuminoid formulation," BMC Complementary and Alternative Medicine, vol. 6, article 10, 2006.

[66] C. La Porte, N. Voduc, G. Zhang et al., "Steady-state pharmacokinetics and tolerability of trans-resveratrol $2000 \mathrm{mg}$ twice daily with food, quercetin and alcohol (Ethanol) in healthy human subjects," Clinical Pharmacokinetics, vol. 49, no. 7, pp. 449-454, 2010.

[67] M. D’Archivio, C. Filesi, R. D. Benedetto, R. Gargiulo, C. Giovannini, and R. Masella, "Polyphenols, dietary sources and bioavailability," Annali dell'Istituto Superiore di Sanità, vol. 43, no. 4, pp. 348-361, 2007.

[68] A. J. Day and G. Williamson, "Biomarkers for exposure to dietary flavonoids: a review of the current evidence for identification of quercetin glycosides in plasma," British Journal of Nutrition, vol. 86, supplement 1, pp. S105-S110, 2001.

[69] K. B. Pandey and S. I. Rizvi, "Plant polyphenols as dietary antioxidants in human health and disease," Oxidative Medicine and Cellular Longevity, vol. 2, no. 5, pp. 270-278, 2009.

[70] K. D. R. Setchell, M. S. Faughnan, T. Avades et al., "Comparing the pharmacokinetics of daidzein and genistein with the use of 13C-labeled tracers in premenopausal women," The American Journal of Clinical Nutrition, vol. 77, no. 2, pp. 411-419, 2003.
[71] B. Wahlstrom and G. Blennow, "A study on the fate of curcumin in the rat," Acta Pharmacologica et Toxicologica, vol. 43, no. 2, pp. 86-92, 1978.

[72] G. Garcea, D. J. Jones, R. Singh et al., "Detection of curcumin and its metabolites in hepatic tissue andportal blood of patients following oral administration," British Journal of Cancer, vol. 90, no. 5, pp. 1011-1015, 2004.

[73] S. I. Hoehle, E. Pfeiffer, A. M. Sólyom, and M. Metzler, "Metabolism of curcuminoids in tissue slices and subcellular fractions from rat liver," Journal of Agricultural and Food Chemistry, vol. 54, no. 3, pp. 756-764, 2006.

[74] G. M. Holder, J. L. Plummer, and A. J. Ryan, "The metabolism and excretion of curcumin (1,7-bis-(4-hydroxy3-methoxyphenyl)-1,6-heptadiene-3,5-dione) in the rat," Xenobiotica, vol. 8, no. 12, pp. 761-768, 1978.

[75] V. Ravindranath and N. Chandrasekhara, "Absorption and tissue distribution of curcumin in rats," Toxicology, vol. 16, no. 3, pp. 259-265, 1980.

[76] M.-H. Pan, T.-M. Huang, and J.-K. Lin, "Biotransformation of curcumin through reduction and glucuronidation in mice," Drug Metabolism \& Disposition, vol. 27, no. 4, pp. 486-494, 1999.

[77] S. K. Vareed, M. Kakarala, M. T. Ruffin et al., "Pharmacokinetics of curcumin conjugate metabolites in healthy human subjects," Cancer Epidemiology Biomarkers \& Prevention, vol. 17, no. 6, pp. 1411-1417, 2008.

[78] E. Wenzel and V. Somoza, "Metabolism and bioavailability of trans-resveratrol," Molecular Nutrition \& Food Research, vol. 49, no. 5, pp. 472-481, 2005.

[79] C. Yu, Y. G. Shin, A. Chow et al., "Human, rat, and mouse metabolism of resveratrol," Pharmaceutical Research, vol. 19, no. 12, pp. 1907-1914, 2002.

[80] D. J. Boocock, G. E. S. Faust, K. R. Patel et al., "Phase I dose escalation pharmacokinetic study in healthy volunteers of resveratrol, a potential cancer chemopreventive agent," Cancer Epidemiology Biomarkers \& Prevention, vol. 16, no. 6, pp. 12461252, 2007.

[81] M. J. Hassett, A. J. O’Malley, J. R. Pakes, J. P. Newhouse, and C. C. Earle, "Frequency and cost of chemotherapy-related serious adverse effects in a population sample of women with breast cancer," Journal of the National Cancer Institute, vol. 98, no. 16, pp. 1108-1117, 2006.

[82] J. Fang, H. Qin, H. Nakamura, K. Tsukigawa, T. Shin, and H. Maeda, "Carbon monoxide, generated by heme oxygenase-1, mediates the enhanced permeability and retention effect in solid tumors," Cancer Science, vol. 103, no. 3, pp. 535-541, 2012.

[83] H. Hashizume, P. Baluk, S. Morikawa et al., "Openings between defective endothelial cells explain tumor vessel leakiness," The American Journal of Pathology, vol. 156, no. 4, pp. 1363-1380, 2000.

[84] K. Iwai, H. Maeda, and T. Konno, "Use of oily contrast medium for selective drug targeting to tumor: enhanced therapeutic effect and X-ray image," Cancer Research, vol. 44, no. 5, pp. 21152121, 1984.

[85] T. Konno, H. Maeda, K. Iwai et al., "Selective targeting of anti-cancer drug and simultaneous image enhancement in solid tumors by arterially administered lipid contrast medium," Cancer, vol. 54, no. 11, pp. 2367-2374, 1984.

[86] A. J. Leu, D. A. Berk, A. Lymboussaki, K. Alitalo, and R. K. Jain, "Absence of functional lymphatics within a murine sarcoma: a molecular and functional evaluation," Cancer Research, vol. 60, no. 16, pp. 4324-4327, 2000. 
[87] B.-H. Jeon, C. Jang, J. Han et al., "Profound but dysfunctional lymphangiogenesis via vascular endothelial growth factor ligands from $\mathrm{CD} 1 \mathrm{~b}^{+}$macrophages in advanced ovarian cancer," Cancer Research, vol. 68, no. 4, pp. 1100-1109, 2008.

[88] T. Safra, F. Muggia, S. Jeffers et al., "Pegylated liposomal doxorubicin (Doxil): reduced clinical cardiotoxicity in patients reaching or exceeding cumulative doses of $500 \mathrm{mg} / \mathrm{m}^{2}$," Annals of Oncology, vol. 11, no. 8, pp. 1029-1033, 2000.

[89] S. Prasad, A. K. Tyagi, and B. B. Aggarwal, "Recent developments in delivery, bioavailability, absorption and metabolism of curcumin: the golden pigment from golden spice," Cancer Research and Treatment, vol. 46, no. 1, pp. 2-18, 2014.

[90] V. Vetvicka, "Glucan-immunostimulant, adjuvant, potential drug," World Journal of Clinical Oncology, vol. 2, no. 2, pp. 115119, 2011.

[91] S. Bisht, G. Feldmann, S. Soni et al., "Polymeric nanoparticleencapsulated curcumin ('nanocurcumin'): a novel strategy for human cancer therapy," Journal of Nanobiotechnology, vol. 5, article 3, 2007.

[92] W. Tiyaboonchai, W. Tungpradit, and P. Plianbangchang, "Formulation and characterization of curcuminoids loaded solid lipid nanoparticles," International Journal of Pharmaceutics, vol. 337, no. 1-2, pp. 299-306, 2007.

[93] L. Guo, Y. Peng, J. Yao, L. Sui, A. Gu, and J. Wang, "Anticancer activity and molecular mechanism of resveratrol-bovine serum albumin nanoparticles on subcutaneously implanted human primary ovarian carcinoma cells in nude mice," Cancer Biotherapy and Radiopharmaceuticals, vol. 25, no. 4, pp. 471-477, 2010.

[94] J. Shao, X. Li, X. Lu et al., "Enhanced growth inhibition effect of resveratrol incorporated into biodegradable nanoparticles against glioma cells is mediated by the induction of intracellular reactive oxygen species levels," Colloids and Surfaces B: Biointerfaces, vol. 72, no. 1, pp. 40-47, 2009.

[95] K. Teskač and J. Kristl, "The evidence for solid lipid nanoparticles mediated cell uptake of resveratrol," International Journal of Pharmaceutics, vol. 390, no. 1, pp. 61-69, 2010.

[96] V. Sanna, I. A. Siddiqui, M. Sechi, and H. Mukhtar, "Resveratrolloaded nanoparticles based on poly(epsiloncaprolactone) and poly(D,L-lactic-co-glycolic acid)-poly(ethylene glycol) blend for prostate cancer treatment," Molecular Pharmaceutics, vol. 10, no. 10, pp. 3871-3881, 2013.

[97] S. S. Darandale and P. R. Vavia, "Cyclodextrin-based nanosponges of curcumin: formulation and physicochemical characterization," Journal of Inclusion Phenomena and Macrocyclic Chemistry, vol. 75, no. 3-4, pp. 315-322, 2013.

[98] F. Trotta and R. Cavalli, "Characterization and applications of new hyper-cross-linked cyclodextrins," Composite Interfaces, vol. 16, no. 1, pp. 39-48, 2009.

[99] F. Trotta, M. Zanetti, and R. Cavalli, "Cyclodextrin-based nanosponges as drug carriers," Beilstein Journal of Organic Chemistry, vol. 8, pp. 2091-2099, 2012.

[100] E. K. Patel and R. J. Oswal, "Nanosponge and microsponges: a Novel Drug Delivery System," International Journal of Research in Pharmacy and Chemistry, vol. 2, no. 2, pp. 2281-2781, 2012.

[101] J. Alongi, M. Poskovic, A. Frache, and F. Trotta, "Role of $\beta$ cyclodextrin nanosponges in polypropylene photooxidation," Carbohydrate Polymers, vol. 86, no. 1, pp. 127-135, 2011.

[102] K. William, S. Benjamin, and H. Eva, "Synthesis and characterization of nanosponges for drug delivery and cancer treatment," December 2011, http://www.vanderbilt.edu/vinse/ docs/William-Kornahrens-Student-Poster.pdf.
[103] S. J. Torne, K. A. Ansari, P. R. Vavia, F. Trotta, and R. Cavalli, "Enhanced oral paclitaxel bioavailability after administration of paclitaxel-loaded nanosponges," Drug Delivery, vol. 17, no. 6, pp. 419-425, 2010.

[104] K. A. Ansari, S. J. Torne, P. R. Vavia, F. Trotta, and R. Cavalli, "Paclitaxel loaded nanosponges: in-vitro characterization and cytotoxicity study on MCF-7 cell line culture," Current Drug Delivery, vol. 8, no. 2, pp. 194-202, 2011.

[105] L. Li, F. S. Braiteh, and R. Kurzrock, "Liposome-encapsulated curcumin," Cancer, vol. 104, no. 6, pp. 1322-1331, 2005.

[106] L. Li, F. S. Braiteh, and R. Kurzrock, "Liposome-encapsulated curcumin: in vitro and in vivo effects on proliferation, apoptosis, signaling, and angiogenesis," Cancer, vol. 104, no. 6, pp. 13221331, 2005.

[107] X.-X. Wang, Y.-B. Li, H.-J. Yao et al., “The use of mitochondrial targeting resveratrol liposomes modified with a dequalinium polyethylene glycol-distearoylphosphatidyl ethanolamine conjugate to induce apoptosis in resistant lung cancer cells," Biomaterials, vol. 32, no. 24, pp. 5673-5687, 2011.

[108] C. Caddeo, K. Teskač, C. Sinico, and J. Kristl, "Effect of resveratrol incorporated in liposomes on proliferation and UVB protection of cells," International Journal of Pharmaceutics, vol. 363, no. 1-2, pp. 183-191, 2008.

[109] D. Suresh and K. Srinivasan, "Tissue distribution \& elimination of capsaicin, piperine \& curcumin following oral intake in rats," Indian Journal of Medical Research, vol. 131, no. 5, pp. 682-691, 2010.

[110] Z. Ma, A. Shayeganpour, D. R. Brocks, A. Lavasanifar, and J. Samuel, "High-performance liquid chromatography analysis of curcumin in rat plasma: application to pharmacokinetics of polymeric micellar formulation of curcumin," Biomedical Chromatography, vol. 21, no. 5, pp. 546-552, 2007.

[111] S. Nunes, R. Madureira, D. Campos et al., "Solid lipid nanoparticles as oral delivery systems of phenolic compounds: overcoming pharmacokinetic limitations for nutraceutical applications," Critical Reviews in Food Science and Nutrition, 2015.

[112] N. K. Narayanan, D. Nargi, C. Randolph, and B. A. Narayanan, "Liposome encapsulation of curcumin and resveratrol in combination reduces prostate cancer incidence in PTEN knockout mice," International Journal of Cancer, vol. 125, no. 1, pp. 1-8, 2009.

[113] F. Figueiró, A. Bernardi, R. L. Frozza et al., "Resveratrol-loaded lipid-core nanocapsules treatment reduces in vitro and in vivo glioma growth," Journal of Biomedical Nanotechnology, vol. 9, no. 3, pp. 516-526, 2013.

[114] K. Cho, X. Wang, S. Nie, Z. Chen, and D. M. Shin, "Therapeutic nanoparticles for drug delivery in cancer," Clinical Cancer Research, vol. 14, no. 5, pp. 1310-1316, 2008.

[115] S. K. Sahoo and V. Labhasetwar, "Enhanced antiproliferative activity of transferrin-conjugated paclitaxel-loaded nanoparticles is mediated via sustained intracellular drug retention," Molecular Pharmaceutics, vol. 2, no. 5, pp. 373-383, 2005.

[116] L. Xu, K. F. Pirollo, W.-H. Tang, A. Rait, and E. H. Chang, "Transferrin-liposome-mediated systemic p53 gene therapy in combination with radiation results in regression of human head and neck cancer xenografts," Human Gene Therapy, vol. 10, no. 18, pp. 2941-2952, 1999.

[117] Z. M. Qian, H. Li, H. Sun, and K. Ho, “Targeted drug delivery via the transferrin receptor-mediated endocytosis pathway," Pharmacological Reviews, vol. 54, no. 4, pp. 561-587, 2002.

[118] M. Mollazade, K. Nejati-Koshki, A. Akbarzadeh et al., "PAMAM dendrimers augment inhibitory effects of curcumin 
on cancer cell proliferation: possible inhibition of telomerase," Asian Pacific Journal of Cancer Prevention, vol. 14, no. 11, pp. 6925-6928, 2013.

[119] H. Tang, C. J. Murphy, B. Zhang et al., "Amphiphilic curcumin conjugate-forming nanoparticles as anticancer prodrug and drug carriers: in vitro and in vivo effects," Nanomedicine, vol. 5, no. 6, pp. 855-865, 2010.

[120] D. J. McClements, Food Emulsions: Principles, Practice and Techniques, CRC Press, Boca Raton, Fla, USA, 2005.

[121] S. Schultz, G. Wagner, K. Urban, and J. Ulrich, "High-pressure homogenization as a process for emulsion formation," Chemical Engineering and Technology, vol. 27, no. 4, pp. 361-368, 2004.

[122] C. J. H. Porter, C. W. Pouton, J. F. Cuine, and W. N. Charman, "Enhancing intestinal drug solubilisation using lipid-based delivery systems," Advanced Drug Delivery Reviews, vol. 60, no. 6, pp. 673-691, 2008.

[123] F. Donsí, Y. Wang, J. I. Li, and Q. Huang, "Preparation of curcumin sub-micrometer dispersions by High-Pressure Homogenization," Journal of Agricultural and Food Chemistry, vol. 58, no. 5, pp. 2848-2853, 2010.

[124] E. Wenzel and V. Somoza, "Metabolism and bioavailability of trans-resveratrol," Molecular Nutrition and Food Research, vol. 49, no. 5, pp. 472-481, 2005.

[125] F. Donsì, M. Sessa, H. Mediouni, A. Mgaidi, and G. Ferrari, "Encapsulation of bioactive compounds in nanoemulsionbased delivery systems," Procedia Food Science, vol. 1, pp. 16661671,2011

[126] S. Mangalathillam, N. S. Rejinold, A. Nair, V.-K. Lakshmanan, S. V. Nair, and R. Jayakumar, "Curcumin loaded chitin nanogels for skin cancer treatment via the transdermal route," Nanoscale, vol. 4, no. 1, pp. 239-250, 2012.

[127] X. Wei, T. H. Senanayake, G. Warren, and S. V. Vinogradov, "Hyaluronic acid-based nanogel-drug conjugates with enhanced anticancer activity designed for the targeting of CD44-positive and drug-resistant tumors," Bioconjugate Chemistry, vol. 24, no. 4, pp. 658-668, 2013.

[128] Q. Du, B. Hu, H.-M. An et al., "Synergistic anticancer effects of curcumin and resveratrol in Hepal-6 hepatocellular carcinoma cells," Oncology Reports, vol. 29, no. 5, pp. 1851-1858, 2013.

[129] L. Masuelli, E. Di Stefano, M. Fantini et al., "Resveratrol potentiates the in vitro and in vivo anti-tumoral effects of curcumin in head and neck carcinomas," Oncotarget, vol. 5, no. 21, pp. 10745-10762, 2014.

[130] P. Mohapatra, S. R. Satapathy, S. Siddharth, D. Das, A. Nayak, and C. N. Kundu, "Resveratrol and curcumin synergistically induces apoptosis in cigarette smoke condensate transformed breast epithelial cells through a $221^{\text {Waf1/Cip1 }}$ mediated inhibition of Hh-Gli signaling," International Journal of Biochemistry and Cell Biology, vol. 66, pp. 75-84, 2015.

[131] K. Coradini, F. O. Lima, C. M. Oliveira et al., "Co-encapsulation of resveratrol and curcumin in lipid-core nanocapsules improves their in vitro antioxidant effects," European Journal of Pharmaceutics and Biopharmaceutics, vol. 88, no. 1, pp. 178-185, 2014.

[132] L. J. Carlson, B. Cote, A. W. Alani, and D. A. Rao, "Polymeric micellar co-delivery of resveratrol and curcumin to mitigate in vitro doxorubicin-induced cardiotoxicity," Journal of Pharmaceutical Sciences, vol. 103, no. 8, pp. 2315-2322, 2014.

[133] K. C. Lund and T. Pantuso, "Combination effects of quercetin, resveratrol and curcumin on in vitro intestinal absorption," Journal of Restorative Medicine, vol. 3, pp. 112-120, 2014. 


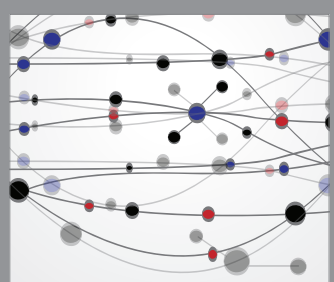

The Scientific World Journal
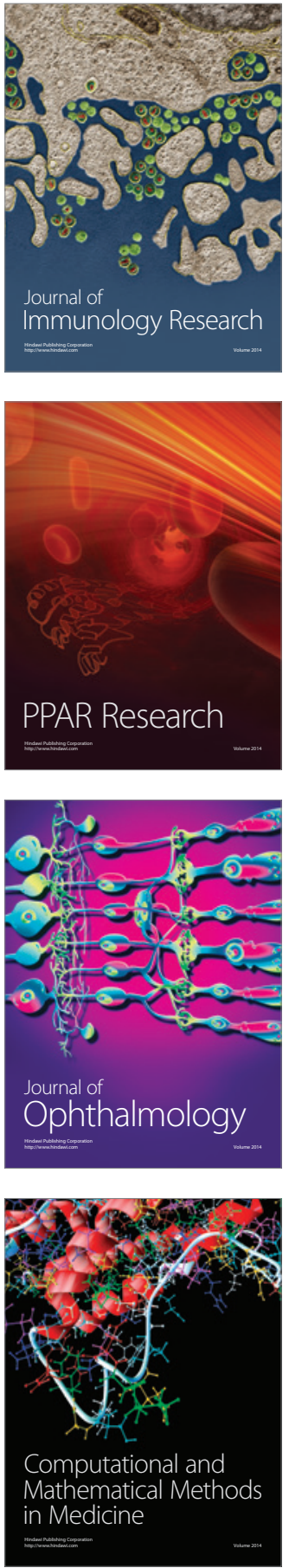

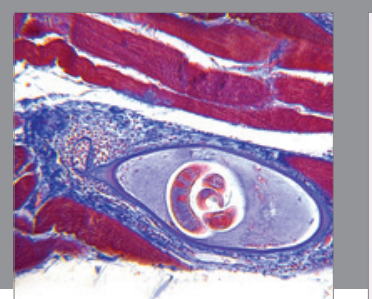

Gastroenterology Research and Practice

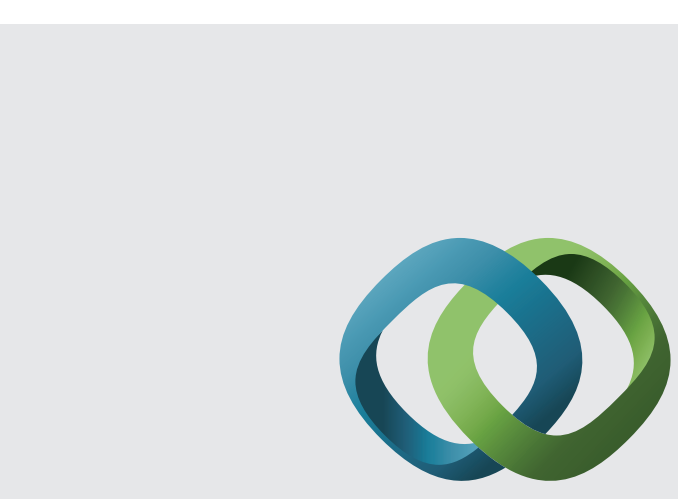

\section{Hindawi}

Submit your manuscripts at

http://www.hindawi.com
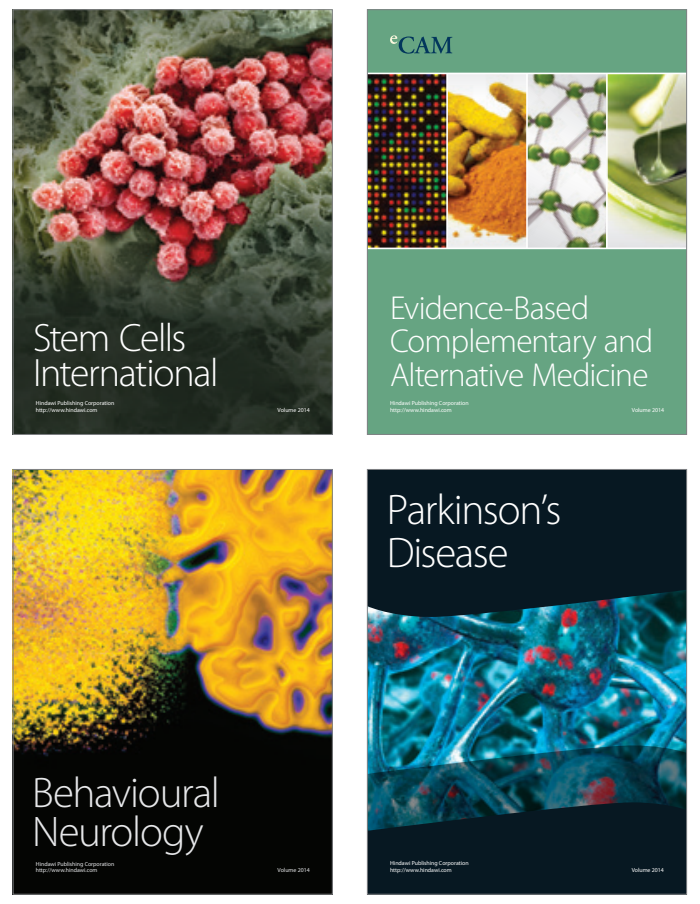
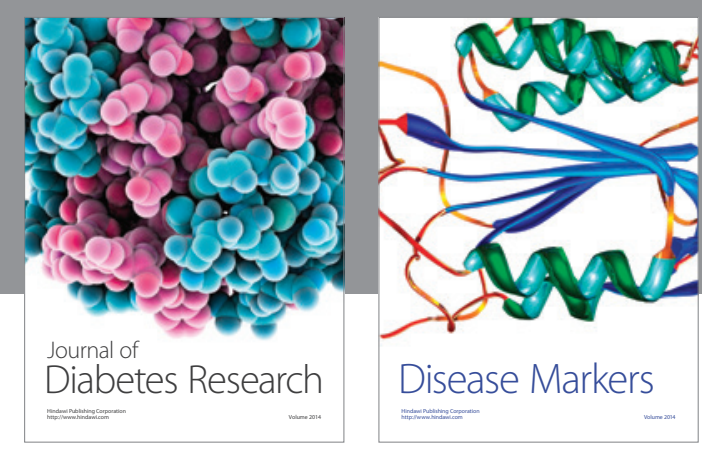

Disease Markers
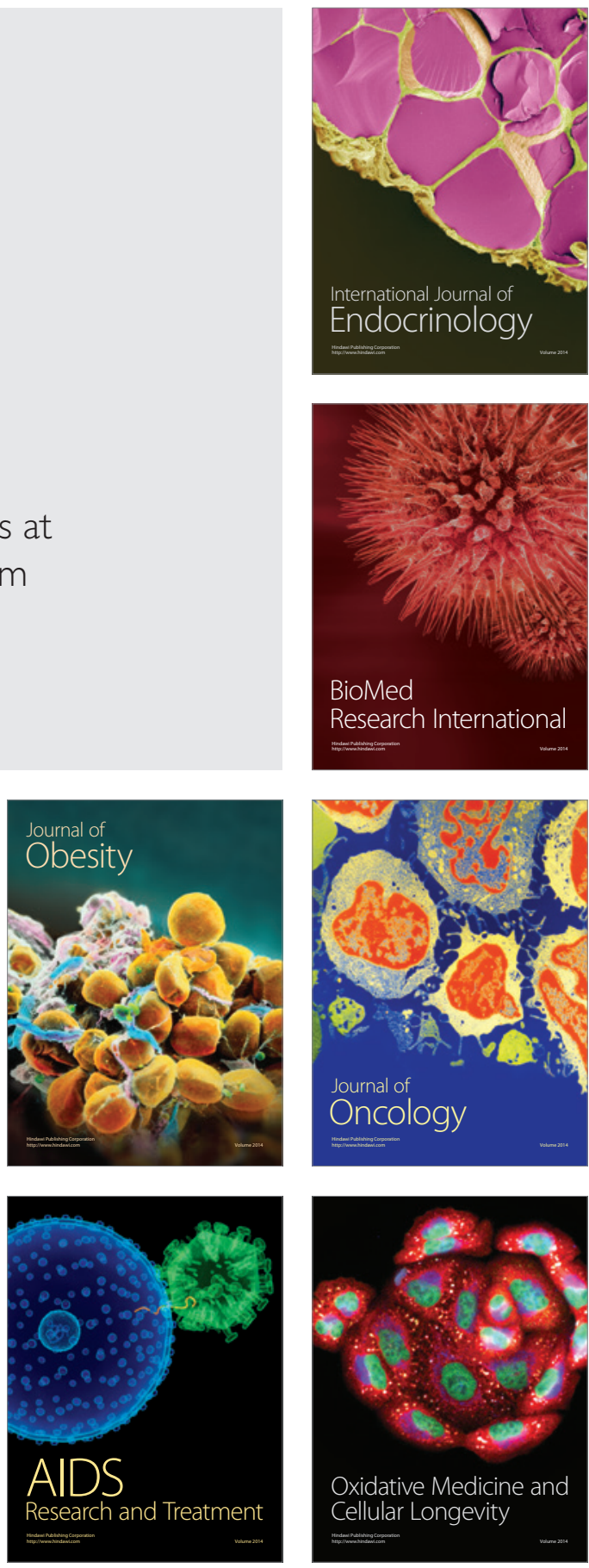